\title{
Adherence and Risk Behaviour in Patients with HIV Infection Receiving Antiretroviral Therapy in Bangkok
}

\author{
Amanda Clarke ${ }^{*}, 1,2$, Stephen Kerr ${ }^{1,2}$, Adam Honeybrook ${ }^{1}$, David A Cooper ${ }^{1,2}$, Anchalee Avihingsanon ${ }^{1}$, \\ Chris Duncombe ${ }^{1}$, Praphan Phanuphak ${ }^{1,3}$, Kiat Ruxrungtham ${ }^{1,3}$, Jintanat Ananworanich ${ }^{1,3}$ and \\ John Kaldor $^{2}$
}

\author{
${ }^{I}$ The HIV Netherlands Australia Thailand Research Collaboration (HIV-NAT), Thai Red Cross AIDS Research Centre, \\ 104 Ratchadamri Road, Bangkok 10330, Thailand \\ ${ }^{2}$ Kirby Institute for Infection and Immunity in Society, University of New South Wales, Sydney NSW 2052, Australia \\ ${ }^{3}$ Department of Medicine, Faculty of Medicine, Chulalongkorn University, Bangkok, 10330, Thailand
}

\begin{abstract}
It could be postulated that due to lifestyle factors, patients with poor antiretroviral therapy (ART) adherence may also have risky sexual behaviour potentially leading to HIV transmission. There are limited data regarding unprotected sex risk and ART adherence in resource limited settings and our study set out to investigate these in an HIV clinic in Bangkok. Patients completed an anonymous questionnaire regarding their relationship details, ART adherence, sexual behaviour, alcohol and drug use and HIV transmission beliefs. Laboratory findings and medical history were also collected. Unprotected sex risk (USR) was defined as inconsistent condom use with a partner of negative or unknown HIV status. Five hundred and twelve patients completed the questionnaire. Fifty seven per cent of patients reported having taken ARV $>95 \%$ of the time in the last month and $58 \%$ had been sexually active in the previous 30 days. Only 27 patients (5\%) were classified as having USR in our cohort. Multivariate analysis showed USR was associated with female gender (OR 2.9, 95\% CI 1.2-7.0, p0.02) but not with adherence, age, type or number of partners, recreational drug or alcohol use nor beliefs about HIV transmission whilst taking ART. Levels of USR in this resource limited setting were reassuringly low and not associated with poor ART adherence; as all USR patients had undetectable viral loads onward HIV transmission risk is likely to be low but not negligible. Nonetheless condom negotiation techniques, particularly in women, may be useful in this group.
\end{abstract}

Keywords: Adherence, Antiretroviral therapy (ART), HIV-1 infection, Unprotected sex risk, Thailand.

\section{INTRODUCTION}

It is well established that adherence to effective antiretroviral treatment (ART) is essential for viral control and hence survival in HIV/AIDS [1]. Earlier studies have shown that ART requires strict dosing schedules for it to be maximally effective, i.e. adherence of $>95 \%$ is needed for optimal viral suppression in patients taking protease inhibitor-based regimens [2]. More recent studies suggest that adherence of $80 \%$ (or even less in some patients) may be sufficient for suppression in the current era of more effective ART, particularly with a longer duration on ART[3, 4].

Having a low serum viral load (VL) is known to be associated with much lower risks of transmission of HIV [5, 6]. However it has been shown that a low plasma viral load does not always correlate with a low genital HIV viral load so transmission could still potentially occur in this situation [7-9]. Yet several studies have shown none or few heterosexual transmissions when a partner is on ART, including a recent randomised controlled international study,

*Address correspondence to this author at the HIV-NAT, The Thai Red Cross AIDS Research Centre, 104 Ratchadamri Road, Pathumwan, Bangkok, 10330, Thailand; Tel: +66 2652 3040; Fax: +66 2252 5779;

E-mail: amanda.c@hivnat.org
HPTN052 showing a $96 \%$ transmission in discordant couples if the infected person takes ART $[10,11]$.

There has been conflicting information regarding the impact of ART use and sexual behaviour: some studies (particularly in resource rich countries) showing increased risk behaviour [12] while others showing a reduction in risk behaviour with ART [13-16] . One study suggested that since ART availability there may be some evidence of increased risky behaviour in non-HIV infected people [17]. Several studies have shown that the belief that having an undetectable viral load leads to lower infectiousness is associated with greater number of partners \& less condom use[18-21] .

Many studies have shown a link between risky sexual behaviour and alcohol and substance abuse use in HIV positive and HIV negative people[22-25]. Some studies have suggested there is a link between poor adherence to ART and increased rates of unprotected sexual intercourse [26, 27], but data from resource limited settings is limited. Poor adherence could be related to erratic lifestyle and health choices; condom use can also be affected by these factors so one could predict the other. Patients with worse adherence are more likely to have detectable VLs, if they then have unsafe sex they could potentially transmit drug-resistant HIV [28]. A study in USA showed an association between poor 
adherence, perceived HIV viral load and increased number of sexual partners [18].

Few studies regarding adherence in Thailand have been reported; one showed good levels [29] but did not assess any correlation with unprotected sex risk. Our study aimed to assess the association in the levels of adherence in chronically infected Thai HIV positive people on stable ART and risky sexual behaviour which could lead to HIV transmission. It also explored use of alcohol, recreational drugs and patients' beliefs regarding HIV transmission and whether these were related to having unprotected sex.

\section{METHODS}

This cross sectional study had ethical approval in both Thailand and Australia. All patients attending a research clinic in Bangkok, who were either on an active clinical trial or on lifelong follow-up post clinical trial and taking ART for at least 6 months between August 2007 and November 2007, were invited to join the study. Participants were asked to fill out an anonymous, self administered questionnaire in Thai regarding their relationship details, ART adherence, sexual behaviour, alcohol and drug use[30]. Adherence in the previous 30 days was assessed using a visual analogue scale (VAS) where patients indicated on a graduated line how often in the past month they took their ART from $0 \%$ (never took it) to $100 \%$ (did not miss a dose). Risk behaviours were assessed using questions adapted from validated questionnaires previously used in Thailand [3133]. This included questions regarding sexual activity in the last 30 days in terms of frequency, numbers/type of partners and condom use. Beliefs regarding HIV transmission were ascertained. A study team member accessed patient notes and computer results system for additional information about medical history and VL/CD4 results closest to date of questionnaire completion. Patients were defined as having unprotected sex risk (USR) if they had any unprotected sex with partners of negative or unknown HIV status in the last 30 days. Patients had no financial compensation for partaking in the study. This study is registered at www.clinicaltrial.gov number NCT00511056.

\section{Statistical Analysis}

Questionnaire responses were entered into a database and patient characteristics were summarised and frequency tables of questionnaire responses were constructed. Whether a patient met our definition of unprotected sex risk (USR) or not was used as the outcome variable in logistic regression models. Covariates assessed for their predictive value were age, gender, education, income, years since HIV diagnosis, years on ART, adherence (dichotomised as good, VAS $>95 \%$, or bad, VAS $<=95 \%$ adherence), type of partner, Viral load results, alcohol \& drug use, belief about HIV transmission in relation to VL or ART. Covariates significant at the level of 0.1 were used to construct a multivariate model. Statistical analysis was conducted using STATA 11.2 (Statacorp, College Station, Tx, USA)

\section{RESULTS}

\section{Demographics}

Five hundred and twelve patients completed the questionnaire and characteristics are summarised in Table $\mathbf{1}$. The median age was 39 years, 286 (56\%) were male. Four hundred and thirty five $(85 \%)$ patients identified themselves heterosexual, 61 (12\%) men who had sex with men (MSM) and $15(3 \%)$ bisexual. The median current CD4 cell count was 511 (IQR 378 - 669) cells $/ \mathrm{mm}^{3} ; 489(96 \%)$ had a $\mathrm{VL}<50$ copies $/ \mathrm{mL}$. Median length of time since HIV diagnosis was 7 (IQR 4 - 9) years and median duration on ART was 5 (IQR $3-7)$ years. Most patients $(71 \%)$ were earning $<10,000$ THB per month (approx 330 USD).

Table 1. Demographics \& HIV Characteristics $(n=512)$

\begin{tabular}{|l|l|}
\hline Gender & $\mathrm{n}, \%$ \\
Male & $286(56)$ \\
female & $226(44)$ \\
\hline Age in years & \\
Median (IQR) & $39(35-45)$, range 24 - 66 \\
\hline Sexuality & $\mathrm{n}, \%$ \\
Heterosexual & $435(85)$ \\
MSM & $61(12)$ \\
Bisexual & $15(3)$ \\
Refused to answer & 1 \\
\hline Education -highest level attained & $\mathrm{n}, \%$ \\
Primary school & $134(26)$ \\
High school & $143(28)$ \\
College & $110(21)$ \\
Bachelor degree & $116(23)$ \\
Masters/doctorate & $7(1.5)$ \\
No answer & $2(0.5)$ \\
\hline Income (Thai baht/month) & $\mathrm{n}, \%$ \\
$<5,000$ & $171(33)$ \\
$5,001-10,000$ & $198(39)$ \\
$10,001-20,000$ & $103(20)$ \\
$>20,000$ & $31(6)$ \\
No answer & $9(2)$ \\
\hline Current CD4 (cells/mm $\left.{ }^{3}\right)$ & \\
Median (IQR) & $511(378-669)$ \\
\hline Viral load, copies/mL & $\mathrm{n}, \%$ \\
$<50$ & $489(96)$ \\
$>50$ \& <200 & $9(2)$ \\
$>200$ & $14(2)$ \\
\hline Years since HIV diagnosis & $5(3-7)$ \\
Median (IQR) & \\
\hline Years of ART & \\
Median (IQR) & \\
\hline Numbers may vary due to missing data. & \\
\hline
\end{tabular}

\section{Partner Information}

Three hundred and forty (66\%) reported having partners: $178(35 \%)$ had a partner who was also HIV positive, 109 (21\%) HIV negative partners and $53(10 \%)$ partners were of unknown HIV status. Two hundred and seventy five $(80 \%)$ had disclosed their HIV status to partners.

\section{ART Adherence}

According to self-reported adherence by VAS, 288 (57\%) patients took $>=95 \%, 178(35 \%)$ took between 80 to 
$<95 \%$ and $42 / 508(8 \%)$ took $<80 \%$ of prescribed doses in the previous month. Eleven out of 14 patients with a VL $>200$ copies $/ \mathrm{mL}$ had a VAS $<95 \%$. The odds ratio for having a detectable VL $>200$ copies $/ \mathrm{mL}$ with an adherence cut point of $<95 \%$ on VAS was $5.0(95 \%$ CI $1.4-18.2)$; $p=0.01$. In the 236 patients who had recently missed dose(s) or taken doses more than 1 hour late, the commonest reasons given were forgetting to take the medicines with them (51\%) and travelling away from home (40\%).

\section{Sexual Behaviour}

In the previous 30 days, 298 (58\%) patients had engaged in sex. For $224(75 \%)$ this was with a regular partner/s and $68(23 \%)$ with a non primary partner ( 8 did not respond).

Most patients $(255,86 \%)$ had had sex with only one partner in the last 30 days, $22(7 \%)$ with 2 partners and 6 patients with 3 or more partners (including one patient with 10 partners). Condoms were consistently used by 240 respondents $(81 \%)$. Reasons given for not using a condom were most commonly due to lack of availability/preparation $(28 \%)$ and the respondent or partner not liking using condoms (34\%). See Table 2.

Table 2. Condom Use and Reasons for Non Use in Last 30 Days in the 298 Sexually Active Patients

\begin{tabular}{|l|l|}
\hline Condom use (n,\%) & \\
Always & $240(81)$ \\
Almost always & $16(5)$ \\
Sometimes & $22(7)$ \\
Never & $18(6)$ \\
No answer given & $2(1)$ \\
\hline Reasons for not using a condom in last 30 days*, n(\%) & \\
Condoms do not feel natural/partner does not like & $26(34)$ \\
Condoms were not available/I did not prepare for using a condom & $21(28)$ \\
Partner also HIV positive so do not use & $14(18)$ \\
Trying to conceive & $5(7)$ \\
Believing that being on HAART will not spread/acquire HIV & $4(5)$ \\
Fear of partner knowing HIV status & $4(5)$ \\
Being drunk & $2(3)$ \\
\hline
\end{tabular}

*Patients could give more than one reason.

\section{Alcohol and Drug Use}

When asked about alcohol intake in the last 30 days, 326 (64\%) had had none, $140(27 \%)$ had alcohol once or less a week, $32(6 \%)$ 2-3 times per week and $9(2 \%)$ had it 4 or more times per week. Sixty-eight patients had alcohol before/during sex in the last 30 days and of these $15(22 \%)$ did not subsequently consistently use a condom. Nine had HIV-positive partners, 5 had HIV-negative/unknown status partners (1 refused to answer).

Recreational drugs were used by $10(2 \%)$ patients, $\leq$ once a week in all. Three used marijuana, 2 benzodiazepines, 1 poppers and the others did not specify what $\operatorname{drug}(\mathrm{s})$ was used. Four of ten $(40 \%)$ used these drugs before or during sex and 2 patients did not always subsequently use condoms. Both of these had $\mathrm{VL}<50$ copies/mL and one had a partner of unknown HIV status.

\section{Patients with Unprotected Sex Risk (USR)}

Twenty seven patients (5\%) fitted the criteria of having a USR, i.e., having any unprotected sex with a partner of negative or unknown HIV status in the last 30 days: $18(67 \%)$ were women; 9 were men (6 heterosexual, 2 MSM, 1 bisexual). All had undetectable viral loads and had been on HAART for at least 2 years. VAS results showed $46 \%$ reported adherence $\geq 95 \%$; this was not significantly different to the non-USR population $(p=0.2)$. Twenty $(77 \%)$ were having sex with their primary partners and 6 with nonprimary/casual partners ( 1 no answer). The commonest reason given for not using a condom was that their partner did not like using condoms $(11,41 \%) ; 9(33 \%)$ said that no condoms were available/they didn't prepare for condom and 2 women did not use a condom for fear of partner discovering status. No USR patients were trying to conceive or believed that being on ART meant they would not spread HIV or were drunk when they had unprotected sex. Seventeen (63\%) patients with USR had disclosed their HIV status to their primary partner. In univariate analysis USR was not associated with poor ART adherence (VAS either $<80$ or $95 \%$ ), alcohol/drug use, casual partners, time on ART or the belief that being on ART prevented transmitting HIV (see Table 3). Younger age (<40years), low education, lower income and female gender were all associated with USR, but after adjustment in a multivariate model, only female gender was significant (odds ratio 2.9; 95\% confidence interval [CI] 1.2 - 7.0; $\mathrm{P}=0.02)$.

\section{Patients' Beliefs Regarding HIV Transmission and HAART}

When asked if taking HIV medicine reduces the threat of transmitting HIV to others 132 (26\%) agreed, $286(56 \%)$ did not and $90(18 \%)$ were unsure. When asked if they thought that having an undetectable viral load ( $<50$ copies) reduces the threat of transmitting HIV to others 99 (19\%) agreed, $294(57 \%)$ disagreed and $114(22 \%)$ were unsure. When asked if taking HIV medicine reduces their concerns about having sex without using a condom 31 (6\%) said yes , 424 $(83 \%)$ said no and $49(10 \%)$ were unsure.

\section{DISCUSSION}

The adherence rates in our cohort were lower than expected with only $57 \%$ reporting adherence of $\geq 95 \%$ in the last 30 days on the visual analogue scale, but despite this the viral loads in our cohort were suppressed in the majority $(96 \%)$ of patients. This finding agrees with other studies noting that the current more potent ART may be more forgiving than previously thought[3]. Unsurprisingly patients reporting a VAS $<95 \%$ had a higher rates of viral loads $>200$ copies $/ \mathrm{mL}$ (OR 5.0). A small number of the patients with very low reported adherence (i.e. $5-45 \%$ on VAS) had undetectable viral loads so miscomprehension of the VAS instructions is a possible explanation although it has previously been well understood in a similar patient group[34]. Conversely some patients with excellent reported adherence had detectable VLs but may have been on failing regimes and waiting for regimen change or resistance results. Pill counts were not done to confirm reported adherence. Reasons for forgetting ART were in the majority practical issues of forgetting to bring the medications with them and 
Table 3. Predictive Factors for Unprotected Sex Risk (USR) in the Previous 30 Days

\begin{tabular}{|l|c|c|c|c|}
\hline \multicolumn{1}{|c|}{ Risk Factor } & \multicolumn{2}{c|}{ Univariate } & \multicolumn{2}{c|}{ Multivariate } \\
\cline { 2 - 5 } & OR (95\% CI) & P & OR (95\% CI) & P \\
\hline \hline Female gender & $4.2(1.8-9.8)$ & 0.001 & $2.9(1.2-7.0)$ & 0.02 \\
\hline Primary school education or less & $2.6(1.1-5.9)$ & 0.02 & $1.9(0.7-4.7)$ & 0.19 \\
\hline Age $<40$ years & $3.2(1.2-8.6)$ & 0.03 & $2.3(0.79-6.5)$ & 0.13 \\
\hline Low income $<5,000 T H B / m o n t h$ & $2.7(1.2-6.1)$ & 0.02 & $1.6(0.65-4.1)$ & 0.29 \\
\hline More than 1 partner in last 30 days & $1.03(0.34-3.15)$ & 0.95 & & \\
\hline Any alcohol in last 30 days & $1.1(0.51-2.47)$ & 0.78 & & \\
\hline Recreational drug use in last 30 days & $3.1(0.62-16.0)$ & 0.17 & & \\
\hline Taking HAART for $\leq 2$ years & $0.97(0.39-2.38)$ & 0.94 & & \\
\hline$<95 \%$ adherence on VAS & $1.6(0.7-3.5)$ & 0.27 & & \\
\hline$<80 \%$ adherence on VAS & $1.4(0.4-5.0)$ & 0.61 & & \\
\hline Sex with primary $(v s$ non-primary) partner & $0.80(0.32-1.90)$ & 0.61 & & \\
\hline Believing transmission is reduced if on HAART \& viral load $<50$ copies $/ \mathrm{mL}$ & $0.98(0.35-2.71)$ & 0.97 & & \\
\hline
\end{tabular}

travelling away from home. This could be potentially be remedied by providing easier more discreet packaging i.e. small pill boxes that can be kept in a pocket/bag at all times.

More than half (58\%) of the patients were sexually active in the last 30 days and in the majority $(80 \%)$ a condom was always used; this rate of condom use is higher than found in cohorts in Kenya (72\%) and Uganda $(66 \%)[35,36]$. In those $56(19 \%)$ who did not always use a condom, more than half had a known HIV positive partner. The risk of an HIV positive person potentially passing on or acquiring another HIV virus appears to be minimal and the larger concern in this small group of seroconcordent couples would be acquiring sexually transmitted diseases and for females of becoming pregnant $[37,38]$. Indeed, trying to conceive was a reason for non condom use in nearly $7 \%$. Information on type of sex was not collected but each type is known to have different risks $[39,40]$. Interestingly nearly $28 \%$ of patients reported the lack of availability and/or lack of planning as a reason for non condom use. Condoms are given out for free to all patients in the HIV clinic so it is more likely that the lack of planning for sexual activity was the cause and patients need to be encouraged to carry condoms on them to be prepared.

Only 27 out of $512(5 \%)$ were classified as USR (having had any unprotected sexual intercourse with a partner of negative or unknown HIV status in the last 30days). This is reassuringly low. The adherence in this group of patients was lower (46 vs 57\%) than non-USR patients but this was not statistically significant and indeed despite this all USR patients had VL $<50$ copies/mL . No information on sexually transmitted diseases was available but a previous study of 824 HIV positive asymptomatic patients in Bangkok showed Chlamydia trachomatis rates of $9.7 \%$ and Neisseria gonorrhoeae of $1.3 \%$, with a high disease burden in sex workers and in younger patients; it is likely our cohort would have lower rates than this with an older median age (39years) and no sex workers [41]. Genital HIV RNA levels were not collected and the true potential infectivity of this small but important group of patients in this cohort is unknown but likely to be very small. Contrary to expectations there was no association of USR and poor adherence in this group. This association may not have been found due to small patient numbers exhibiting USR but may indicate that the unprotected sex was not due to 'erratic' lifestyles but rather due to other sex-related factors such as condom issues and HIV disclosure concerns. Indeed $40 \%$ reported their partner's dislike of condoms as the reason for unprotected sexual intercourse. This exemplifies the complex nature of sexual risks including the importance of condom negotiation skills, particularly for women (who made up $67 \%$ of USR). Female gender was the only factor associated with increased risk of USR (OR 2.9). Ragnarsson et al., recently reported a similar finding in a cohort in Kenya with female gender having an OR of 3.03 for inconsistent condom use [35]. This group suggested possible reasons for their finding being a lack of individual negotiating power in intimate relationships and also reproductive desires; in our study none of the USR women reported reproductive desire as their reason for non condom use so difficulty with condom negotiation or partner choice are likely factors in our cohort and further work needs to be done e.g. acceptability testing of other barrier methods such as the female condom and teaching condom negotiation techniques.

In the questions regarding beliefs only $26 \%$ patients thought that taking ART would reduce transmission of HIV yet even fewer patients believed that having an undetectable viral load would affect HIV transmission. This study was carried out when there was less evidence regarding ART and HIV transmission, also many of the patients in the cohort have low incomes and may not have access to the latest HIV news e.g. through the internet and networks, to guide their decision making in unprotected sex risk. Medical staff discussing latest scientific information regarding transmission with patients need to clearly state the evidence so far and carefully tailor their advice to a patient's 
individual situation to ensure partners are not inadvertently put at risk.

Only a small proportion of patients reported recreational drug use and over $2 / 3$ did not drink alcohol; this implies that this cohort is not a high 'risk taking' group, either because of self selection by being someone willing to enter into a clinical trial or due to the regular counselling and advice given regarding risk behaviours, or likely a combination of both these factors. Of those who drank alcohol around the time of sex $22 \%$ did not subsequently use a condom and this supports findings of many other studies showing alcohol intake can lead to subsequent unsafe sexual practises[23, 24].

Limitations of this study include the fact that this was performed in a research setting so results may not be transferable to the general population where less intense medical input and counselling related to sexual risk and adherence may occur. Patients were only asked about sexual activity in the last 30 days which may have underestimated true sexual activity and hence risk behaviour in the cohort. The number of MSM in the cohort was low reflecting the epidemic of HIV in Thailand being largely heterosexual.

In summary unprotected sex risk was uncommon $(27 / 512,5 \%)$ and poor ART adherence was not associated with USR in this resource limited cohort. Women were significantly more likely to have USR and more work needs to be done to help women in condom negotiation particularly in serodiscordant relationships. HIV prevention strategies need to be focused on high transmission risk groups to be maximally effective (i.e. patients not on ART, seroconverting patients, undiagnosed patients) and this outpatient clinic cohort appears to have a reassuring low, although not negligible, risk of transmission.

\section{ACKNOWLEDGEMENT}

Declared none.

\section{CONFLICTS OF INTEREST}

Declared none.

\section{REFERENCES}

[1] Wood E, Hogg RS, Yip B, Harrigan PR, O'Shaughnessy MV, Montaner JS. Effect of medication adherence on survival of HIVinfected adults who start highly active antiretroviral therapy when the CD4+ cell count is 0.200 to $0.350 \times 10(9)$ cells/L. Ann Intern Med 2003; 139: 810-6.

[2] Paterson DL, Swindells S, Mohr J, et al. Adherence to protease inhibitor therapy and outcomes in patients with HIV infection. Ann Intern Med 2000; 133: 21-30.

[3] Bangsberg DR. Less than 95\% adherence to nonnucleoside reversetranscriptase inhibitor therapy can lead to viral suppression. Clin Infect Dis 2006; 43: 939-41.

[4] Rosenblum M, Deeks SG, van der Laan M, Bangsberg DR. The risk of virologic failure decreases with duration of HIV suppression, at greater than $50 \%$ adherence to antiretroviral therapy. PLoS One 2009; 4: e7196.

[5] Quinn TC, Wawer MJ, Sewankambo N, et al. Viral load and heterosexual transmission of human immunodeficiency virus type 1. Rakai Project Study Group. N Engl J Med 2000; 342: 921-9.

[6] Donnell D, Baeten JM, Kiarie J, et al. Heterosexual HIV-1 transmission after initiation of antiretroviral therapy: a prospective cohort analysis. Lancet 2010; 375: 2092-8.

[7] $\mathrm{Cu}-U$ vin S, DeLong AK, Venkatesh KK, et al. Genital tract HIV-1 RNA shedding among women with below detectable plasma viral load. AIDS 2010; 24: 2489-97.
[8] Marcelin AG, Tubiana R, Lambert-Niclot S, et al. Detection of HIV-1 RNA in seminal plasma samples from treated patients with undetectable HIV-1 RNA in blood plasma. AIDS 2008; 22: 1677-9.

[9] Halfon P, Giorgetti C, Khiri H, et al. Semen may harbor HIV despite effective HAART: another piece in the puzzle. PLoS One 2010; 5: e10569.

[10] Attia S, Egger M, Muller M, Zwahlen M, Low N. Sexual transmission of HIV according to viral load and antiretroviral therapy: systematic review and meta-analysis. AIDS 2009; 23: 1397-404.

[11] Cohen MS, Chen YQ, McCauley M, et al. Prevention of HIV-1 Infection with Early Antiretroviral Therapy. N Engl J Med 2011; 365: 493-505.

[12] Stolte IG, de Wit JB, van Eeden A, Coutinho RA, Dukers NH. Perceived viral load, but not actual HIV-1-RNA load, is associated with sexual risk behaviour among HIV-infected homosexual men. AIDS 2004; 18: 1943-9.

[13] Bunnell R, Ekwaru JP, Solberg P, et al. Changes in sexual behavior and risk of HIV transmission after antiretroviral therapy and prevention interventions in rural Uganda. AIDS 2006; 20: 85-92.

[14] Peltzer K, Ramlagan S. Safer sexual behaviours after 1 year of antiretroviral treatment in KwaZulu-Natal, South Africa: a prospective cohort study. Sex Health 2010; 7: 135-41.

[15] Eisele TP, Mathews C, Chopra M, et al. Changes in risk behavior among HIV-positive patients during their first year of antiretroviral therapy in Cape Town South Africa. AIDS Behav 2009; 13: 1097105 .

[16] Kennedy C, O'Reilly K, Medley A, Sweat M. The impact of HIV treatment on risk behaviour in developing countries: a systematic review. AIDS Care 2007; 19: 707-20.

[17] Shafer LA, Nsubuga RN, White R, et al. Antiretroviral therapy and sexual behavior in Uganda: a cohort study. AIDS 2011; 25: 671-8.

[18] Kalichman SC, Cherry C, Amaral CM, et al. Adherence to antiretroviral therapy and HIV transmission risks: implications for test-and-treat approaches to HIV prevention. AIDS Patient Care STDS 2010; 24: 271-7.

[19] Joseph HA, Flores SA, Parsons JT, Purcell DW. Beliefs about transmission risk and vulnerability, treatment adherence, and sexual risk behavior among a sample of HIV-positive men who have sex with men. AIDS Care 2010; 22: 29-39.

[20] Seng R, Rolland M, Beck-Wirth G, et al. Trends in unsafe sex and influence of viral load among patients followed since primary HIV infection, 2000-2009. AIDS 2011; 25: 977-88.

[21] Crepaz N, Hart TA, Marks G. Highly active antiretroviral therapy and sexual risk behavior: a meta-analytic review. JAMA 2004; 292: 224-36.

[22] Van Tieu H, Koblin BA. HIV, alcohol, and noninjection drug use. Curr Opin HIV AIDS 2009; 4: 314-8.

[23] Woolf SE, Maisto SA. Alcohol use and risk of HIV infection among men who have sex with men. AIDS Behav 2009; 13: 75782 .

[24] Mansergh G, Flores S, Koblin B, Hudson S, McKirnan D, Colfax GN. Alcohol and drug use in the context of anal sex and other factors associated with sexually transmitted infections: results from a multi-city study of high-risk men who have sex with men in the USA. Sex Transm Infect 2008; 84: 509-11.

[25] Beckett M, Burnam A, Collins RL, Kanouse DE, Beckman R. Substance use and high-risk sex among people with HIV: a comparison across exposure groups. AIDS Behav 2003; 7: 209-19.

[26] Wilson TE, Barron Y, Cohen M, et al. Adherence to antiretroviral therapy and its association with sexual behavior in a national sample of women with human immunodeficiency virus. Clin Infect Dis 2002; 34: 529-34.

[27] Kalichman SC, Rompa D. HIV treatment adherence and unprotected sex practices in people receiving antiretroviral therapy. Sex Transm Infect 2003; 79: 59-61.

[28] Goldsamt LA, Clatts MC, Parker MM, Colon V, Hallack R, Messina MG. Prevalence of Sexually Acquired Antiretroviral Drug Resistance in a Community Sample of HIV-Positive Men Who Have Sex with Men in New York City. AIDS Patient Care STDS 2011; 25: 287-93.

[29] Maneesriwongul WL, Tulathong S, Fennie KP, Williams AB. Adherence to antiretroviral medication among HIV-positive patients in Thailand. J Acquir Immune Defic Syndr 2006; 43 Suppl 1: S119-22. 
[30] Mannheimer SB, Mukherjee R, Hirschhorn LR, et al. The CASE adherence index: A novel method for measuring adherence to antiretroviral therapy. AIDS Care 2006; 18: 853-61.

[31] Allen DR, Carey JW, Manopaiboon C, et al. Sexual health risks among young Thai women: implications for HIV/STD prevention and contraception. AIDS Behav 2003; 7: 9-21.

[32] Liu A, Kilmarx P, Jenkins RA, et al. Sexual initiation, substance use, and sexual behavior and knowledge among vocational students in northern Thailand. Int Fam Plan Perspect 2006; 32: 126-35.

[33] Mansergh G, Naorat S, Jommaroeng R, et al. Inconsistent condom use with steady and casual partners and associated factors among sexually-active men who have sex with men in Bangkok, Thailand. AIDS Behav 2006; 10: 743-51.

[34] Kerr SJ, Avihingsanon A, Pucharoen O, et al. Assessing adherence in Thai patients taking HAART. Int J STD AIDS 2012 (in press).

[35] Ragnarsson A, Ekstrom AM, Carter J, et al. Sexual risk taking among patients on antiretroviral therapy in an urban informal settlement in Kenya: a cross-sectional survey. J Int AIDS Soc 2011; 14: 20.

[36] Wagner GJ, Holloway I, Ghosh-Dastidar B, Ryan G, Kityo C, Mugyenyi P. Factors associated with condom use among HIV clients in stable relationships with partners at varying risk for HIV in Uganda. AIDS Behav 2010; 14: 1055-65.

[37] Ssemwanga D, Lyagoba F, Ndembi N, et al. Multiple HIV-1 infections with evidence of recombination in heterosexual partnerships in a low risk Rural Clinical Cohort in Uganda. Virology 2011; 411: 113-31.

[38] Smith DM, Richman DD, Little SJ. HIV superinfection. J Infect Dis 2005; 192: 438-44.

[39] Vittinghoff E, Douglas J, Judson F, McKirnan D, MacQueen K, Buchbinder SP. Per-contact risk of human immunodeficiency virus transmission between male sexual partners. Am J Epidemiol 1999; 150: 306-11.

[40] Gray RH, Wawer MJ, Brookmeyer R, et al. Probability of HIV-1 transmission per coital act in monogamous, heterosexual, HIV-1discordant couples in Rakai, Uganda. Lancet 2001; 357: 1149-53.

[41] Srifeungfung S, Roongpisuthipong A, Asavapiriyanont S, et al. Prevalence of Chlamydia trachomatis and Neisseria gonorrhoeae in HIV-seropositive patients and gonococcal antimicrobial susceptibility: an update in Thailand. Jpn J Infect Dis 2009; 62: 467-70.

(C) Clarke et al.; Licensee Bentham Open.

This is an open access article licensed under the terms of the Creative Commons Attribution Non-Commercial License (http: //creativecommons.org/licenses/by$\mathrm{nc} / 3.0 /$ ) which permits unrestricted, non-commercial use, distribution and reproduction in any medium, provided the work is properly cited. 